\title{
A Striking Similarity Between the Sun, Binary Systems and RR Lyrae Stars
}

\author{
V. A. Kotov ${ }^{1,2}$ \\ ${ }^{1}$ Crimean Astrophysical Observatory, Crimea, USSR \\ ${ }^{2}$ Stanford University, Stanford CA 94305 U.S.A.
}

\begin{abstract}
The periodicity of $P_{0}=160.0101( \pm 1)$ min discovered in the Sun's global oscillations, then in rapid variability of active galactic nuclei (AGNs) (Kotov \& Lyuty 1990), might have a cosmological origin. Analysing the distribution of periods of $\sim 4000$ close binaries we found that the most commensurate (with an odd-even parity taken into account) period of galactic binaries is equal to $160.0( \pm 1) \min (\sim 4 \sigma)$. Accordingly, it is argued that the famous $2-3 \mathrm{hr}$ gap of cataclysmic binaries might be closely associated with the "universal" $P_{0^{-}}$ oscillation. A similar analysis applied to the $\sim 1200 \mathrm{RR}$ Lyrae variables in galactic globular clusters (GCs) and 72 RR Lyraes in the LMC GCs (Graham 1985; Nemec et al. 1985) showed that for all those stars the $P_{0}$-period appears to be the most "resonant" one (Fig. 1). This strongly supports the cosmological origin of the $P_{0}$-oscillation. Since the $P_{0}$-resonance for RR Lyraes appears to be most pronounced when the "odd-even" parity is taken into account, it is hypothesized that variability in the majority of GC RR Lyraes should be related to their binary nature and may be relevant to explanation of Blazhko effect.
\end{abstract}

\section{References:}

Graham, J. 1985, PASP, 97, 676.

Kotov, V.A. \& Lyuty, V.M. 1990, Compt. Rend. Acad. Sci. Paris, 310, 743.

Nemec, J.M., Hesser, J.E. \& Ugarte, P.P. 1985, ApJS, 57, 287.
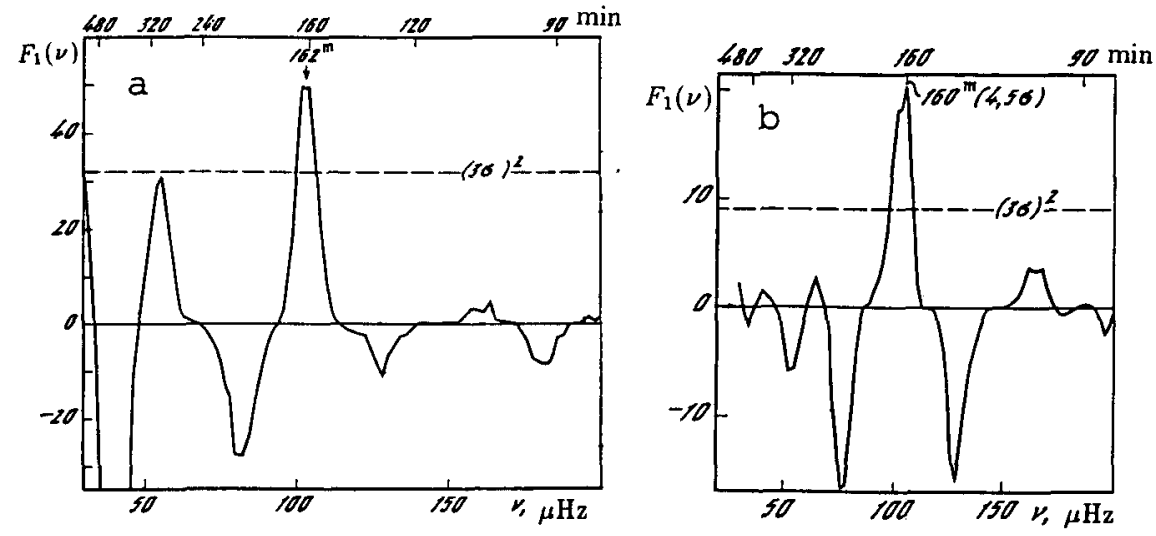

Fig. 1. The "resonance spectra computed for (a) 1211 RR Lyrae stars in galactic globular clusters, and (b) 72 RR Lyrae stars discovered in the LMC globular clusters. 